\title{
Dried Distillers Grains Supplemented at Different Frequencies to Stocker Heifers Grazing Late-Season Flint Hills Native Pastures
}

\author{
M.P. Epp, D.A. Blasi, W.L. Metzen, and B.E. Oleen
}

\section{Introduction}

Wetter-than-normal summer grazing seasons can produce excessive forage beyond what the typical Flint Hills double-stock harvest rate can remove. These late-summer native grasses do not contain adequate protein to sustain economical gains for stocker cattle, but producers can extend the grazing season by using a protein-based supplement to generate economically feasible rates of gain. Use of dried distillers grains as a supplementation program can help promote overall productivity during the fall grazing season. However, the drawback is the potential high labor input for supplement delivery. Delivering supplements at reduced frequencies per week may be able to sustain daily gains of stocker cattle while reducing labor costs associated with supplement delivery.

\section{Experimental Procedures}

One 72-day grazing period was conducted at the Kansas State University Beef Stocker Unit starting September 1, 2009, to determine the response to various frequencies of dried distillers grains supplementation of heifers grazing Flint Hills native grasses. All cattle were sourced from a local sale barn 12 days prior to the study, vaccinated for clostridial and respiratory diseases, dewormed, and fed a high-roughage diet free choice. To obtain a more uniform group of animals, cattle with extreme high or low weights or cattle that exhibited signs of prior illness, lameness, or poor disposition were removed from the pool of cattle eligible for the experiment.

Cattle were randomized and stratified by weight to obtain an equal stocking rate per paddock. The stocking rate per paddock was approximately $63 \mathrm{lb} /$ acre. There were three treatment groups and three replications (paddocks) per treatment group. All cattle were fed dried distillers grains at a daily rate of $0.33 \%$ body weight (dry matter basis), but the dried distillers grains were delivered at one of three frequencies: every day, every other day, or every third day. On the basis of predicted gains of $1.5 \mathrm{lb} /$ head daily, dried distillers grains supplementation was increased every 2 weeks during the grazing period. All cattle were hand-fed in bunks located in each pasture. Forage samples were collected at three different times during the grazing study from four different pastures and submitted to a commercial laboratory for nutrient analysis.

Performance data were analyzed by using the MIXED model procedure of SAS (SAS Institute, Inc., Cary, NC). Data were arranged in a randomized complete block design, and pasture was the experimental unit for growth outcomes as affected by treatment. In the model, fixed effects were treatment and pasture, and random effects were pasture $x$ treatment, pasture, and animal. 


\section{Results and Discussion}

Average daily gain was not different among the treatment groups (Table 1). Cattle fed dried distillers grains every other day or every third day gained at essentially the same rate as cattle supplemented daily during the 72 -day grazing period that was initiated in September 2009.

Crude protein and energy content of the clipped forage collected from the four paddocks declined at a marked rate over this study (Table 2).

\section{Implications}

If adequate grass resources are available in late summer, producers can reduce labor costs by supplementing dried distillers grains to cattle every second or third day and still achieve performance that is similar to feeding dried distillers grains daily.

Table 1. Performance of stocker heifers fed dried distillers grains (DDG) at a daily rate of $0.33 \%$ of body weight (dry basis) at different frequencies while grazing Flint Hills native grasses in late summer and fall

\begin{tabular}{lccccc}
\hline & \multicolumn{3}{c}{ DDG supplementation frequency } & & \\
\cline { 2 - 4 } Item & Daily & $\begin{array}{c}\text { Every other } \\
\text { day }\end{array}$ & $\begin{array}{c}\text { Every third } \\
\text { day }\end{array}$ & SEM & P-value \\
\hline No. of pastures & 3 & 3 & 3 & & \\
No. of cattle & 17 & 17 & 17 & & \\
DDG $^{1}$, total lb fed & 508 & 507 & 501 & & \\
Initial weight, lb & 620 & 622 & 614 & 8.7 & $>0.54$ \\
Final weight, lb & 727 & 728 & 715 & 10.1 & $>0.38$ \\
Average daily gain, lb & 1.51 & 1.49 & 1.43 & 0.15 & $>0.71$ \\
\hline
\end{tabular}

${ }^{1}$ DDG nutrient content: $90 \%$ dry matter, $30 \%$ crude protein. 
Table 2. Fall 2009 nutritional quality of native pastures at the Kansas State University Beef Stocker Unit

\begin{tabular}{|c|c|c|c|c|c|c|c|c|c|c|c|c|}
\hline \multirow[b]{3}{*}{ Item } & \multicolumn{3}{|c|}{ Pasture 3} & \multicolumn{3}{|c|}{ Pasture 7} & \multicolumn{3}{|c|}{ Pasture 13} & \multicolumn{3}{|c|}{ Pasture 17} \\
\hline & \multicolumn{3}{|c|}{ Sampling date } & \multicolumn{3}{|c|}{ Sampling date } & \multicolumn{3}{|c|}{ Sampling date } & \multicolumn{3}{|c|}{ Sampling date } \\
\hline & $8 / 31$ & $9 / 29$ & $11 / 2$ & $8 / 31$ & $9 / 29$ & $11 / 2$ & $8 / 31$ & $9 / 29$ & $11 / 2$ & $8 / 31$ & $9 / 29$ & $11 / 2$ \\
\hline \multicolumn{13}{|l|}{ Nutrient composition } \\
\hline Dry matter, \% & 38.3 & 36.3 & 73.4 & 44.3 & 40.4 & 72.9 & 43.0 & 44.2 & 80.27 & 41.2 & 43.9 & 77.3 \\
\hline Crude protein, $\%$ & 6.8 & 4.9 & 3.1 & 5.3 & 4.7 & 3.1 & 5.3 & 4.7 & 3.04 & 6.3 & 4.2 & 2.5 \\
\hline Soluble crude protein, $\%$ of crude protein & 28.8 & 25.0 & 25.0 & 40.6 & 25.0 & 25.0 & 25.0 & 25.0 & 25.0 & 38.3 & 25.0 & 25.0 \\
\hline Fat, \% & 2.4 & 2.5 & 2.1 & 1.8 & 2.4 & 2.5 & 1.9 & 1.8 & 2.5 & 2.4 & 1.9 & 2.3 \\
\hline Acid detergent fiber, $\%$ & 36.0 & 36.4 & 40.4 & 34.3 & 36.4 & 41.1 & 33.8 & 37.5 & 37.7 & 36.0 & 40.3 & 42.0 \\
\hline Neutral detergent fiber, $\%$ & 64.6 & 62.2 & 68.2 & 62.4 & 62.1 & 69.3 & 61.4 & 63.9 & 64.1 & 62.3 & 68.3 & 70.7 \\
\hline \multicolumn{13}{|l|}{ Calculated analysis } \\
\hline Net energy maintenance, Mcal/cwt & 58.0 & 52.3 & 49.6 & 50.8 & 51.9 & 46.5 & 52.7 & 47.3 & 49.2 & 56.9 & 50.0 & 49.6 \\
\hline Net energy gain, $\mathrm{Mcal} / \mathrm{cwt}$ & 32.1 & 26.8 & 24.4 & 25.5 & 26.5 & 21.5 & 27.2 & 22.2 & 24.0 & 31.1 & 24.2 & 24.4 \\
\hline Total digestible nutrients, $\%$ & 59.0 & 55.3 & 53.6 & 54.4 & 55.1 & 51.6 & 55.6 & 52.1 & 53.3 & 58.3 & 53.9 & 53.6 \\
\hline Calcium , \% & 0.38 & 0.82 & 0.43 & 0.47 & 1.03 & 0.97 & 0.83 & 0.84 & 1.24 & 0.56 & 0.47 & 0.47 \\
\hline Phosphorus, \% & 0.12 & 0.07 & 0.04 & 0.10 & 0.13 & 0.06 & 0.09 & 0.07 & 0.04 & 0.11 & 0.08 & 0.05 \\
\hline Magnesium, \% & 0.20 & 0.23 & 0.14 & 0.24 & 0.24 & 0.17 & 0.10 & 0.09 & 0.11 & 0.23 & 0.19 & 0.14 \\
\hline Potassium, \% & 1.16 & 0.69 & 0.11 & 1.12 & 0.65 & 0.25 & 0.83 & 0.67 & 0.12 & 1.09 & 0.66 & 0.19 \\
\hline Sulfur, \% & 0.12 & 0.08 & 0.05 & 0.11 & 0.09 & 0.07 & 0.08 & 0.07 & 0.07 & 0.10 & 0.07 & 0.05 \\
\hline Iron, ppm & 178.57 & 209 & 153 & 234.29 & 291 & 263 & 238.37 & 216 & 175 & --- & 174 & 118 \\
\hline Manganese, ppm & 41.29 & 38 & 36 & 16.51 & 17 & 21 & 37.15 & 49 & 53 & --- & 32 & 40 \\
\hline Copper, ppm & 11.35 & 14 & 11 & 11.35 & 12 & 10 & 11.35 & 13 & 13 & -- & 13 & 10 \\
\hline Zinc, ppm & 29.93 & 38 & 34 & 32.00 & 35 & 43 & 28.89 & 33 & 39 & --- & 30 & 30 \\
\hline Molybdenum, ppm & --- & 1 & 1 & 3.10 & 2 & 1 & 1.03 & 1 & 1 & --- & 1 & 1 \\
\hline Cobalt, ppm & --- & 1 & 1 & 3.10 & 1 & 1 & --- & 1 & 1 & --- & 1 & 1 \\
\hline Selenium, ppm & --- & 0.05 & 0.04 & --- & 0.03 & 0.03 & --- & 0.02 & 0.03 & --- & --- & 0.03 \\
\hline
\end{tabular}

